\title{
Energy in ICT - Trends and Research Directions
}

\author{
Ward Van Heddeghem, Willem Vereecken, Mario Pickavet and Piet Demeester ${ }^{1}$
}

\begin{abstract}
Power consumption of ICT equipment has a growing impact, both on economic and environmental level. To reduce its energy footprint, drastic actions will be required on four fronts: energy-efficient components, power management techniques, new network paradigms and policy supporting actions. This paper outlines the current trends and research for each category.
\end{abstract}

Index Terms-power consumption, internet, electricity, network concept

\section{INTRODUCTION}

The energy consumption of Information and Communication Technology (ICT) equipment is estimated to be around $4 \%$ of the global primary energy consumption in 2008, and this percentage is projected to double by 2020 [1]. It is clear that the pressure on reduction of the energy footprint of ICT has become more and more prominent, for mainly two reasons:

- Economic - the total cost of ownership of network exchanges is rising both because higher capacity network equipment becomes more power hungry and requires more cooling, as well as due to increasing energy prices.

- Environmental - the alarming reports on global climate change have fueled the research for reducing the carbon footprint of all human activities, including ICT equipment.

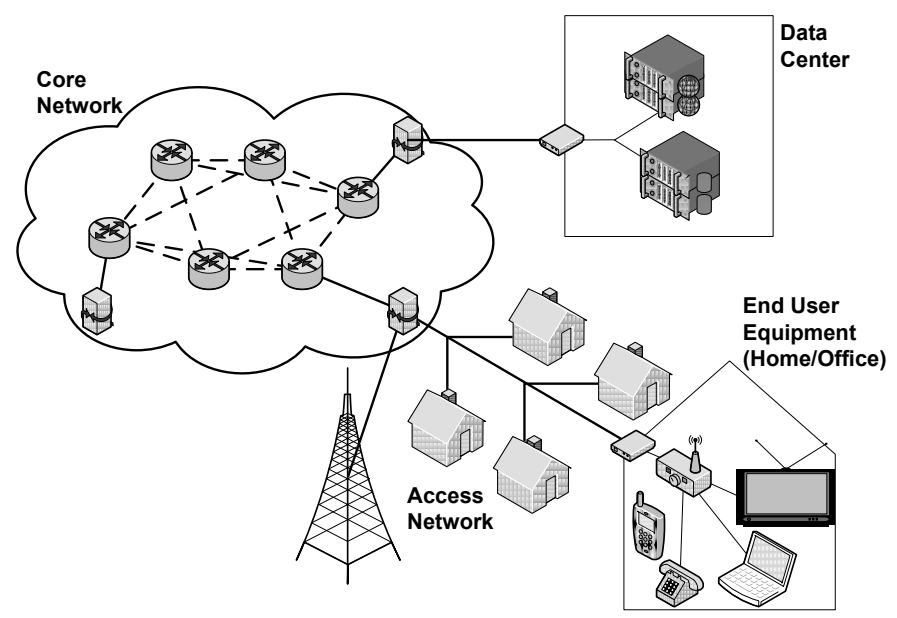

Fig. 1 - Communication network and attached terminal devices

ICT equipment comprises not only traditional equipment like network devices, servers and PCs, but also consumer electronics attached to the communication network such as

\footnotetext{
${ }^{1}$ Manuscript submitted on October $31^{\text {st }}, 2009$. The authors are with the Department of Information Technology (INTEC) of Ghent University - IBBT, Gaston Crommenlaan 8, B-9050 Gent, Belgium (e-mail: \{firstname.lastname\}@intec.ugent.be).
}

TVs, DVD players and gaming consoles. Fig. 1 provides an overview of what we consider ICT equipment in this paper.

Reducing the energy footprint of ICT significantly requires initiatives on a number of fronts: (a) improving energyefficiency of hardware components, (b) better power management of devices and technologies, (c) switching to new network paradigms, and (d) adapting policy supporting actions. We will illustrate the current trends and research for each of these categories in the following sections.

In the remainder of this paper, we will concentrate on energy consumption of ICT during its use phase; we will not consider life cycle assessment (LCA) which in addition includes energy consumption during excavation, production, transport and end-of-life.

\section{ENERGY-EFFICIENT COMPONENTS}

One of the key measures is the power optimization of individual devices. From [1], it is clear that the ICT footprint on power consumption will not be dominated by one equipment type: servers, PCs, network equipment and TVs are all representing a considerable share of the overall footprint. Diverse measures will be needed to improve the overall situation drastically, ranging for instance from more powerefficient chip design for CPUs to better technologies for TV and computer screens. Consider the comparison of laptop and desktop PCs, where the power consumption of a laptop PC is typically only a small fraction of a desktop PC, for basically the same functionality. Another example is research towards more energy-efficient set-top boxes [2], which become more and more widespread with the advance of digital and highdefinition audio and video content.

Energy-proportional computing [3] makes a case for improving the energy usage profile at various operation loads.

\section{Power Management Techniques}

Next to reducing the energy consumption of the devices, it is also important to make sure they only consume power when required. Two key ideas govern energy-saving through power management: (a) exploiting idle periods of activity to temporarily put the device(s) in a low-power sleep state, and (b) slowing down to consume less energy when maximum performance is not required.

\section{A. Network}

When considering layer 2 (link layer) network links, several power management techniques are possible. The IEEE Study Group on Energy-Efficient Ethernet [4] explores the 
realization of Adaptive Link Rate (ALR), allowing to temporarily reduce the bandwidth and hence also the power consumption of Ethernet links during low traffic periods and to return quickly to full bandwidth when needed. A backwards compatible variation on ALR that is proposed by the same study group is PAUSE Power Cycle [5]. A LAN switch periodically pauses all active links connected to it and powers off during the times the links are paused, effectively reducing the link rate.

Most current network protocols make a sleeping PC lose its network connectivity, effectively preventing the PC from entering a low-power sleeping state. One general approach to maintain network presence is for a PC (or any networked product) to transfer network presence to a "proxy" when entering sleep mode [6]. The proxy can be the PC's built-in network interface card or an external device such as the LAN switch.

Individual protocols can be modified as well. Green BitTorrent [7] is a proposed adaptation of the standard BitTorrent protocol that allows the client to sleep when not upor downloading, potentially reducing the client power consumption by $75 \%$.

In the core network, multilayer traffic engineering, which originally optimizes backbone topologies for costs, has been studied to optimize for low energy consumption [8]. Using this technique, low-load links (typically with high idle power consumption) are disabled and traffic is migrated to other links.

\section{B. Data Center}

In data centers the technique of virtualization can realize significant power savings. Virtualization can be applied on two levels. Firstly, multiple instances can be shared on one physical server, reducing the number of servers and thus the power consumption. Secondly, scalable systems using multiple physical servers can be built allowing to switch off most servers during periods of low use and only using the full capacity of servers during peak hours.

A simulation study on grid power management for a large French experimental grid platform showed that automatically turning off idle grid nodes allowed power savings of up to $45 \%$ [9].

In overlay networks, such as peer-to-peer file-sharing networks or Voice-over-IP networks (e.g., Skype), dynamic node activation can be used to selectively deactivate a subset of the overlay nodes at times of low network load [10].

\section{End User}

Although software is only indirectly linked with powerefficiency, its impact can be considerable, especially when it is widely used.

For example, given Microsoft's dominant usage share on the PC operating system market, Windows 7's power management improvements can have a huge overall impact. Improvements include automatic integrated display dimming, focusing on idle activity by removing or coalescing background activity to enter low power states more frequently, allowing for central control of power management settings in companies, and support for network presence proxying [11][12].

\section{NEW NETWORK PARADIGMS}

Whereas the previous measures concentrate typically on one type of equipment, it is crucial to consider also the big picture of various terminals all around the world interconnected via a common network infrastructure. New, clean-slate network paradigms could change the energy footprint of ICT drastically, leading to lower power consumption for both network infrastructure and terminal devices.

\section{A. Network}

Demand for high-transmission capacity is expected to increase considerably in the near future. However, power consumption is expected to become a main limiting factor for scaling the current high-capacity network nodes up to these projected even higher capacities. A study on future highcapacity nodes [13] states that the use of optical technology in high-capacity switches and routers is generally more energy-efficient than electronic technology. Also, circuitswitched nodes consume less than packet-switched architectures, being $90 \%$ for the optical case, and $43 \%$ for the electronic case. The study concludes that a kind of hybrid switching that efficiently combines packet, circuit, and burst switching within the core network would lead to a lower complexity of core nodes and to a significant reduction in total power consumption.

A study by Baldi and Ofek [14] proposes the energy-saving and energy-scaling potential of a circuit-switching technique called pipeline forwarding to transport predictable internet traffic in a more energy-efficient way on a separate network.

\section{B. Data Center}

Next to optimizing the power consumption of ICT it is also important to enable renewable energy sources - such as solar, wind or hydro power - to supply power to ICT systems. This approach seems specifically applicable to data centers, which can be located at renewable energy production sites. However, since renewable energy sources tend to be unpredictable (e.g., wind), or vary during day and night (e.g., solar power), this would imply that the data itself needs to be migrated from one datacenter to the other, following a so-called follow-the-sun or chase-the-wind scenario. As a consequence, energy-efficient high bandwidth networks and routing architectures will be required.

Along this line of thought, a study performed by Kien Le et al. [15] investigates cost-aware and energy-aware load distribution across multiple data centers. The study evaluates the potential cost and carbon savings for data centers located in different time zones and partly powdered by green energy. When optimizing for green energy use, green data centers can decrease $\mathrm{CO} 2$ emission by $35 \%$ by leveraging the green data centers at only a $3 \%$ cost increase. 


\section{End User}

In the 'Thin-Client' concept [16] the key idea is to outsource most or all of the processing and storage tasks from a regular PC client to servers. This allows replacing the PC by a much smaller and simpler client machine (the thin client) that is communicating frequently with the servers via the network. For higher server share ratios (i.e., the amount of clients handled by a server), up to $66 \%$ of the desktop power consumption can be saved by replacing it with a thin client setup.

\section{POLICY SUPPORTING ACTIONS}

Estimating the energy and environmental footprint of alternative ICT solutions is a key element to define clear future goals. This holds both from the viewpoint of an ICT equipment vendor, who wants to distinguish his product from the crowd by emphasizing the low power costs and green characteristics of the product, and from the viewpoint of a government or regulatory body, that wants to promote environmentally friendly products through legislation, financial stimulation, product labels, etc.

However, estimating the footprint accurately is in many cases a highly complex problem. Examples of initiatives and standardization in this area are the Power Usage Effectiveness (PUE) and Energy Consumption Rating (ECR) metrics. The PUE [17] expresses the energy efficiency of data centers and is the ratio of the total power consumed by a data center to the power consumed by the IT equipment that populates the facility. ECR [18], targeted towards high-end packet-based network and telecom equipment, defines a testing methodology and expresses the energy consumption per maximum throughput, typically Watt/Gbps. ECRW is a similar weighted metric that also takes into account off-peak and idle conditions.

The Energy Star label is a well-established international energy-rating label for electronic consumer products. Other examples of power consumption guidelines include the European Union's Code of Conducts that target ICT (related) equipment, such data centers, digital TV services, and broadband communication equipment. Fig. 2 shows the evolution of power consumption per port guidelines for a number of access network technologies as defined in each update of the Code of Conduct on Energy Consumption of Broadband Communication Equipment [19].

\section{ACKNOWLEDGMENTS}

This research is supported by the BONE project ("Building the Future Optical Network in Europe"), a Network of Excellence funded by the European Community's Seventh Framework, and by the IBBT-project GreenICT.

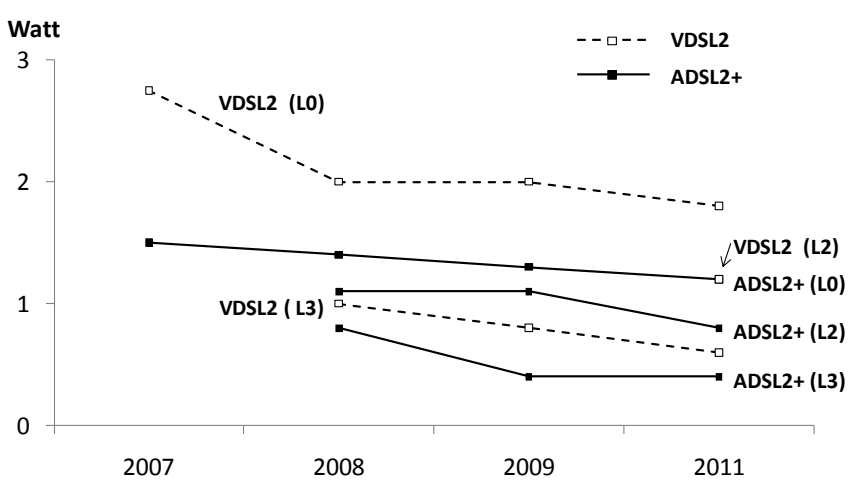

Fig. 2 - Evolution of power consumption guidelines for xDSL access network port values $(\mathrm{L} 0=$ full on, $\mathrm{L} 2=$ low power, $\mathrm{L} 3=\mathrm{idle})$.

\section{REFERENCES}

[1] M. Pickavet et al, "Worldwide Energy Needs for ICT: the Rise of Power-Aware Networking," IEEE ANTS 2008, Bombay (India): 2008.

[2] The Energy-Efficient Set-top Box, http://efficientnetworks.lbl.gov/enetboxes.html

[3] L.A. Barroso and U. Hölzle, "The Case for Energy-Proportional Computing," IEEE Computer, vol. 40, 2007, pp. 33-37.

[4] IEEE 802.3 Study Group on Energy-Efficient Ethernet, http://www.ethernetalliance.org

[5] F. Blanquicet and K. Christensen, "PAUSE Power Cycle: A New Backwards Compatible Method to Reduce Energy Use of Ethernet Switches", ethernet alliance, 2008.

[6] B. Nordman and K. Christensen, "Improving the Energy Efficiency of Ethernet-Connected: A Proposal for Proxying", ethernet alliance, 2007.

[7] J. Blackburn and K. Christensen, "A Simulation Study of a New Green BitTorrent," Proceedings of the First International Workshop on Green Communications, Dresden (Germany), 2009.

[8] B. Puype et al., "Energy Efficient Multilayer Traffic Engineering," Proc 35th European Conference on Optical Communication (ECOC 2009), Vienna (Austria), 2009, pp. 24-25.

[9] A. Orgerie et al., "Save Watts in your Grid: Green Strategies for EnergyAware Framework in Large Scale Distributed Systems," 14th IEEE International Conference on Parallel and Distributed Systems (ICPADS'08), Melbourne (Australia), 2008, pp. 171-178.

[10] J. Famaey et al., "Dynamic Overlay Node Activation Algorithms for Large-Scale Service Deployments," Lecture Notes in Computer Science, vol. 5273, 2008, pp. 14-27.

[11] Microsoft Corporation, white Paper: "Power Management in Windows 7 Overview", May 2009, retrieved from http://www.microsoft.com/environment/windows7.aspx, on 2009-10-22

[12] Microsoft Corporation, presentation: "Network Power Management Fundamentals", retrieved from http://www.microsoft.com/environment/windows7.aspx, on 2009-10-22

[13] A. Slaviša, "Analysis of Power Consumption in Future High-Capacity Network Nodes," Journal of Optical Communications and Networking (JOCN), vol. 1, 2009, pp. 245-258.

[14] M. Baldi and Y. Ofek, "Time for a "Greener" Internet," Communications Workshops, 2009. ICC Workshops 2009. IEEE International Conference on, Dresden (Germany), 2009, pp. 1-6.

[15] K. Le et al., "Cost- and Energy-Aware Load Distribution Across Data Centers," SOSP Workshop on Power Aware Computing and Systems (HotPower '09), Big Sky Montana (USA): 2009.

[16] W. Vereecken et al., "Power Efficiency of Thin Clients", submitted to European Transactions on Telecommunications, Sept. 2008.

[17] The Green Grid, "The Green Grid Data Center Power Efficiency Metrics: PUE and DCiE," Technical Committee White Paper, 2008

[18] The Energy Consumption Rating (ECR) initiative, $\mathrm{http}: / /$ www.ecrinitiative.org/

[19] European Commission, "Code of Conduct on Energy Consumption of Broadband Equipment (Version 3)," 2008, http://re.jrc.ec.europa.eu/energyefficiency/html/standby_initiative_broa dband\%20communication.htm. 